\title{
Flight Test Results for Uniquely Tailored Propulsion- Airframe Aeroacoustic Chevrons: Shockcell Noise
}

\author{
Vinod G. Mengle, ${ }^{*}$ Ulrich W. Ganz, ${ }^{\dagger}$ Eric Nesbitt, ${ }^{\ddagger}$ and Eric J. Bultemeier ${ }^{\S}$ \\ The Boeing Company, Seattle, WA, 98124-2207 \\ and \\ Russell H. Thomas ${ }^{* *}$ \\ NASA Langley Research Center, Hampton, VA 23681-2199
}

\begin{abstract}
Azimuthally varying chevrons (AVC) which have been uniquely tailored to account for the asymmetric propulsion-airframe aeroacoustic interactions have recently shown significant reductions in jet-related community noise at low-speed take-off conditions in scale model tests of coaxial nozzles with high bypass ratio. There were indications that such AVCs may also provide shockcell noise reductions at high cruise speeds. This paper describes the flight test results when one such AVC concept, namely, the T-fan chevrons with enhanced mixing near the pylon, was tested at full-scale on a modern large twin-jet aircraft (777-300ER) with focus on shockcell noise at mid-cruise conditions. Shockcell noise is part of the interior cabin noise at cruise conditions and its reduction is useful from the viewpoint of passenger comfort. Noise reduction at the source, in the exhaust jet, especially, at low frequencies, is beneficial from the perspective of reduced fuselage sidewall acoustic lining. Results are shown in terms of unsteady pressure spectra both on the exterior surface of the fuselage at several axial stations and also microphone arrays placed inside the fuselage aft of the engine. The benefits of T-fan chevrons, with and without conventional chevrons on the core nozzle, are shown for several engine operating conditions at cruise involving supersonic fan stream and subsonic or supersonic core stream. The T-fan AVC alone provides up to $5 \mathrm{~dB}$ low-frequency noise reduction on the fuselage exterior skin and up to 2 dB reduction inside the cabin. Addition of core chevrons appears to increase the higher frequency noise. This flight test result with the previous model test observation that the Tfan AVCs have hardly any cruise thrust coefficient loss $(<0.05 \%)$ make them viable candidates for reducing interior cabin noise in high bypass ratio engines.
\end{abstract}

\section{Nomenclature}

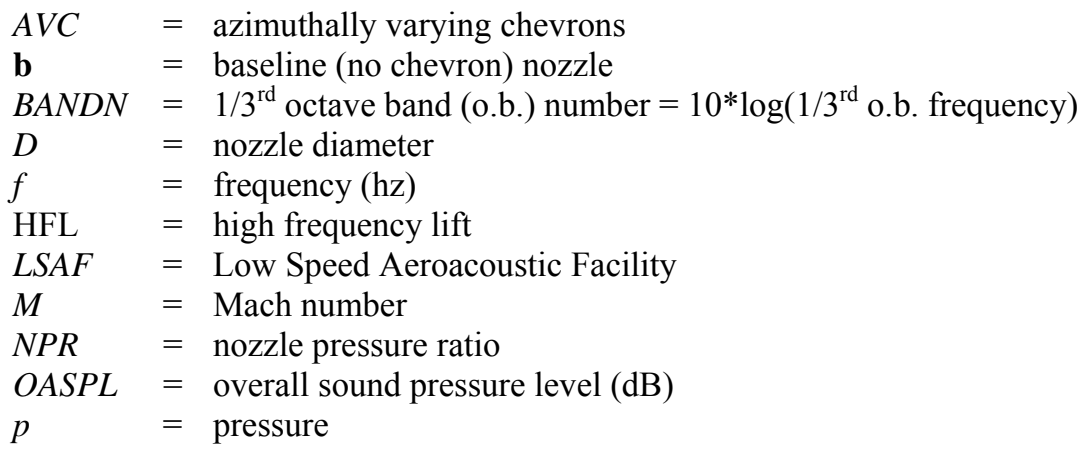

* Engineer/Scientist, Acoustics \& Fluid Mechanics Dept., PO Box 3707, MC:67-ML. Senior Member, AIAA.

${ }^{\dagger}$ Boeing Associate Technical Fellow, Acoustics \& Fluid Mechanics Dept., PO Box 3707, MC:67-ML

$\$$ Boeing Associate Technical Fellow, Noise Product Development, PO Box 3707 M/S OR-MM. Member, AIAA.

$\S$ Engineer, Acoustics \& Fluid Mechanics Dept., PO Box 3707, MC:67-ML. Member, AIAA.

${ }^{* *}$ Senior Research Engineer, Aeroacoustics Branch, MS 166. Senior Member, AIAA. 


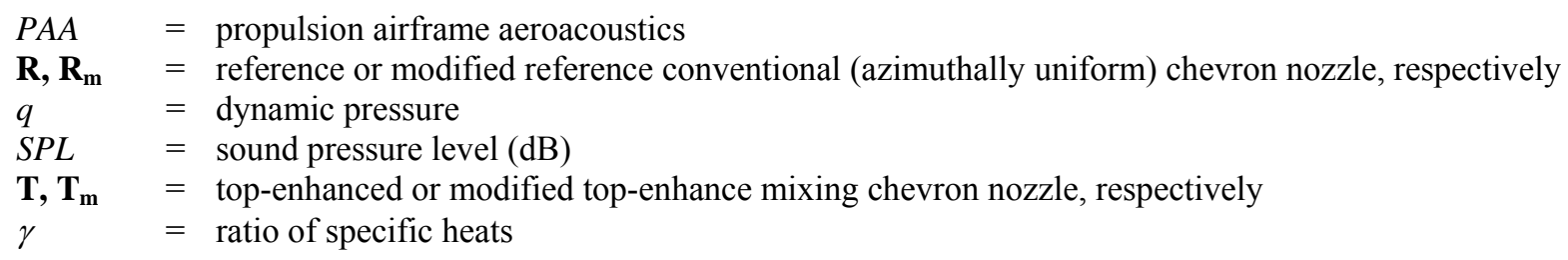

$\begin{array}{ll}\text { Subscripts } \\ a m b \quad=\text { ambient } \\ b l & =\text { boundary layer } \\ m & =\text { modified } \\ p & =\text { primary flow } \\ s & =\text { secondary flow } \\ s c n & =\text { shockcell noise } \\ w t & =\text { wind-tunnel }\end{array}$

\section{Introduction}

W HEN an engine is installed on an airframe in the conventional engine-under-the-wing configuration, the flow/acoustic environment becomes highly asymmetric due to the pylon, the upwash and the noise reflection from the wing, the asymmetric interaction of the jet exhaust with the trailing edge of the wing or high-lift devices installed on it, etc. These constitute the propulsion-airframe aeroacoustic (PAA) interactions. The conventional state-of-the-art chevrons, pioneered in Janardan et al. ${ }^{1}$ with a goal of reducing jet-mixing noise at take-off conditions, are azimuthally uniform serrations on the core and/or fan nozzle lip, and have not addressed this PAA issue due to asymmetry. Recently, in a series of papers, Mengle et al., ${ }^{2,4}$ have introduced the concept of azimuthally varying chevrons (AVC) to take advantage of this PAA installation asymmetry and discovered through a series of systematic scale-model tests in an open-jet wind-tunnel that certain AVCs give larger noise benefits in the far field compared to conventional chevrons for modern high-bypass-ratio operating conditions at take-off. In particular, the uniquely tailored T-fan chevron nozzle, with enhanced mixing near the pylon and less mixing diametrically away from it, when combined with conventional core chevrons, showed the highest far field noise reductions at take-off conditions. Interestingly, the T-fan AVC gave good low frequency noise reduction with hardly any high frequency lift which is endemic to conventional chevrons. Figure 1, taken from Mengle et al., shows the unique T-fan chevron nozzle in conjunction with the regular or R-chevron core nozzle (or RT-nozzle for short) and also in comparison to the conventional or regular chevrons on both fan and core nozzle (RR-nozzle).

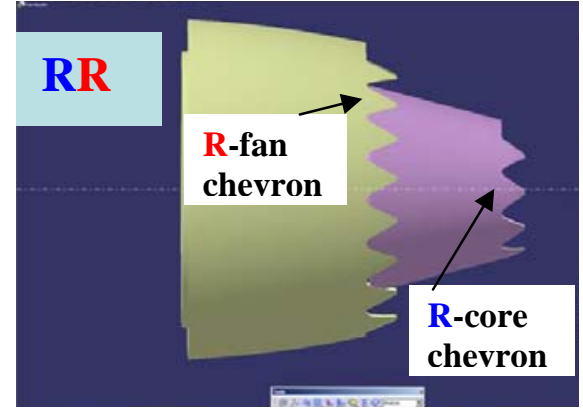

(a) RR-nozzle

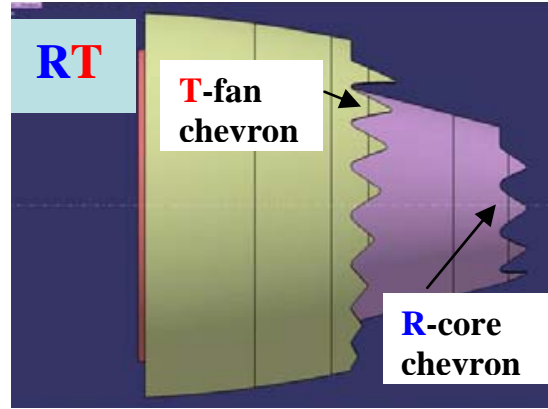

(b) RT-nozzle

Figure 1. Azimuthally varying chevrons (AVC) with top-enhanced mixing on fan nozzle (T-fan chevrons) in comparison to the conventional (R) uniform chevrons: (a) RR-nozzle (b) RTnozzle.

With this success of the T-fan chevrons in model-scale tests, we decided to test that concept at full-scale in a flight test under Boeing's Quiet Technology Demonstrator 2 program. QTD2 is a Boeing-led partnership between Boeing, GE, NASA, Goodrich and Japan's ANA to test the feasibility of several noise reduction concepts in a flight 
test and whose overview is given in Herkes et al. ${ }^{5}$ A companion paper by Nesbitt et al. ${ }^{6}$ discusses the flight test results for community noise at take-off conditions for these T-fan chevrons. This paper, on the other hand, focuses on the shockcell noise results at cruise conditions, important from the viewpoint of interior cabin noise.

More stringent regulations relative to the noise exposure of flight crews and increased expectations by passengers for a quiet cabin environment make it necessary for aircraft manufacturers to consider interior cabin noise in their design requirements. Reduced cabin noise levels can be achieved by either increasing the transmission loss through the fuselage sidewall or by reducing levels generated by the noise sources. Increasing the transmission loss at the fuselage sidewall is typically associated with an increase in the weight of the sidewall. This is undesirable since it will result in a reduction of the revenue-generating payload.

This paper is concerned with the interior noise in the aft cabin (aft of the wing trailing edge) of large commercial aircraft during cruise conditions. The primary noise sources that affect this area are the boundary layer noise and the noise generated by the engine jet plumes. The boundary layer noise results from the unsteady pressure field associated with the turbulence convecting in the boundary layer of the fuselage. In the cruise condition, the exhaust jet flows are supersonic and imperfectly expanded. As a result of this broadband shockcell noise becomes the dominant noise component generated by the jets. Shockcell noise results from the interaction of the turbulence convecting in the shear layers with the quasi-stationary shockcell structure in the jet plume. The turbulence is governed by the shear between adjacent streams.

In typical commercial aircraft engines there are two shear layers: the outer between the secondary nozzle flow and the ambient flow field, and the inner shear layer between jet flows emerging from the primary and the secondary nozzles. The shockcell structure is primarily a function of the Mach number of the jet flows and the nozzle geometry. The Mach numbers of the exhaust flows are controlled by the nozzle pressure ratios defined as the ratio between the total pressure of the exhaust flow and the ambient static pressure. The nozzle pressure ratios in turn can be represented as the product of the ram pressure ratio defined as the ratio between the total and static pressures of the ambient flow field and an engine total pressure ratio which is a function of the engine power setting. In the case of the secondary flow this internal pressure ratio is very close to the total pressure ratio across the fan stage. In typical turbofan engines of commercial aircraft the internal total pressure ratio is higher for the secondary than for the primary flow.

Also in today's single stage turbofan engines, the nozzle pressure ratios are subcritical for aircraft operation at low forward speeds. Supercritical nozzle operation occurs when both the ram pressure ratio and the engine power setting are high. This occurs during aircraft climb and cruise conditions, when the flight Mach numbers exceed 0.6 and when thrust requirements are high, such as when the aircraft climbs to a new cruise altitude or during the initial phase of a cruise segment. During supercritical nozzle operation the exhaust flows are imperfectly expanded and since the nozzles typically only include a very small divergent section, the flows are underexpanded. As a result of this the flow expands downstream of the nozzle beyond its perfectly expanded state and initiates a standing wave pattern in the exhaust plume. This standing wave pattern also referred to as shockcells will interact with the wavelike disturbances convecting in the shear layers to generate shockcell noise.

Shockcell noise has been studied for many decades. An early fundamental study was done by Harper-Bourne and Fisher ${ }^{7}$. They demonstrated that the peak of shockcell noise is associated with the constructive interference between the noise emitted from adjacent shockcells and developed a prediction procedure for shockcell noise of single flow supersonic jets from convergent nozzles. Tam and Tanna ${ }^{8}$ extended the prediction of shockcell noise to convergent-divergent nozzle geometries. Tam, in Ref. 8 and numerous other papers, developed a shockcell noise prediction method for single flow, imperfectly expanded jets based on the interaction of large scale structures convected in the shear layer with the waveguide modes of the shockcell structure. Norum and Shearin ${ }^{9}$ reported shockcell static pressure and noise measurements at freestream Mach numbers from 0.0 to 0.4. Their results indicate that the peaks associated with the higher order waveguide modes become more pronounced with increasing freestream Mach number. Bhat et al. ${ }^{10}$ documented model scale shockcell noise measurements in the Boeing Low Speed Aeroacoustic Facility and showed that shockcell noise resulting from a dual flow co-annular jet rig does not monotonically increase with jet Mach number but is a complex function of the Mach numbers of the two jets.

The measurement of shockcell noise in high speed facilities that are capable of simulating freestream Mach numbers typical for aircraft cruise conditions is difficult because of their high noise floors and because of the reverberant properties of the test sections. Long ${ }^{11}$ used phased microphone array technique to extract the shockcell noise generated by a model scale jet rig from the total signals measured in a transonic test facility.

In the following sections, we first show some model-scale test results at cruise gas conditions which motivated us to do full-scale flight test at cruise with such AVC nozzles, then we describe the flight test aircraft, nozzles, and instrumentation, and briefly describe the test procedure and data analysis procedure for the exterior unsteady pressure measurements and interior sound measurements. Finally, we present the flight test results for the T-fan 
nozzle alone and then in combination with the conventional core chevron nozzle and compare them to the baseline nozzle results. Some parametric variations with engine cycle conditions are also presented.

\section{Model Scale Results at "Pseudo-Cruise" Conditions}

During the model scale tests ${ }^{2,3,4}$ with AVCs for studying take-off and approach conditions, mentioned earlier, we also tested a few cases with gas conditions representative of mid-cruise conditions with the fan stream supercritical and the core stream subcritical, but with the open-jet wind-tunnel at only a Mach number, $\mathrm{M}_{\mathrm{wt}}$, of 0.32 , not the typical cruise value of 0.84 or so of a modern jet aircraft. This is the maximum Mach number which is attainable with the jet rig in Boeing's LSAF. Compared to noise generated at actual cruise conditions and received on the fuselage this leads to several limitations, some of which are enumerated below:

a) LSAF $\mathrm{M}_{\mathrm{wt}}=0.32$ instead of $\mathrm{M}_{\mathrm{a} / \mathrm{c}}=0.84$

b) LSAF wind-tunnel noise at high Mach numbers can contaminate the acoustic signal

c) LSAF secondary temperature is greater than actual engine secondary temperature at cruise conditions (which is much cooler at cruise altitudes) but at least the core to fan temperature ratio can be kept the same

d) LSAF isolated nozzle versus real installed nozzle under the wing

e) LSAF nozzle has no core vent geometry or vent flow, whereas, the test engine has a core vent with flow (tertiary flow)

f) LSAF nozzle fan and core chevrons were slightly different than the actual ones on the test engine.

g) LSAF observer is "underneath" the pylon, whereas the real observer (in or on the fuselage) is "orthogonal" to the pylon.

h) LSAF acoustic data is in the far field instead of near field data collected on the fuselage.

The first limitation is the strongest one because the wind-tunnel Mach number governs the spreading rate of the fan/ambient shear layer which, in turn, governs the shockcell spacing in the fan stream and the frequency of shockcell noise. It also affects the convective speed of eddies in the shear layer, as well as the convection of rays from the source (the jet) to the observer, and, hence, the polar directivity pattern. The relative contribution of jet noise to shockcell noise will also be vastly different at such different ambient speeds. Hence, we will call these conditions as "pseudo-cruise" conditions even if the nozzle pressure ratios and temperature ratios can be matched with the desired ones at cruise conditions.

Knowing these limitations on the use of data collected from LSAF at pseudo-cruise conditions it is still instructive to see the difference that AVCs make in the far field sound compared to that from conventional chevrons and the baseline nozzle. Part of the flow-acoustic mechanism which assists the T-fan chevron nozzle to reduce jetmixing noise better than the conventional R-chevron nozzle at take-off conditions may still be operative at higher jet speeds and affect the shockcell noise. It is only with this expectation that we tested the scale model at pseudo-cruise conditions, not with any anticipation that it will match the flight test results at cruise.

Figure 2 shows a comparison of the far field SPL results for the isolated baseline nozzle (bb), the nozzle with conventional chevrons on both fan and core nozzles (RR), and the nozzle with T-fan chevrons and conventional Rcore chevrons (RT), all with a pylon, at "pseudo=cruise" conditions of $\mathrm{NPR}_{\mathrm{s}}=2.33$ and $\mathrm{NPR}_{\mathrm{p}}=1.74$. This gives supersonic secondary (or fan) stream and subsonic primary (or core) stream, which is representative of mid-cruise conditions. Thus, we expect shock-cells to form in the fan stream and create shockcell noise, besides the usual jetmixing noise. Note the very low frequency data in Fig. 2 (band $<25$ or so) is attributable to wind-tunnel noise and should be neglected.

Figure 2(a) shows a very interesting observation: in the angular range of $70^{\circ}$ to $120^{\circ}$, the conventional RR-nozzle shifts the SPL spectra to higher frequencies compared to the baseline bb-nozzle without changing the peak SPL value much, whereas, the RT-nozzle, with the T-fan chevrons, shifts the SPL spectra to the right on the frequency axis but at the same time reduces the peak SPL considerably. This shift of the SPL spectra for the RT-nozzle to the right and down leads to a difference in SPL compared to the baseline nozzle, as shown in Fig. 2(b): a large reduction in SPL at low frequencies, and simultaneous high frequency lift (HFL) in SPL. The large SPL reduction of above 3 $\mathrm{dB}$ at aft angles for low frequencies must be the usual reduction in jet mixing noise, as also seen in previous studies at take-off conditions; the remaining SPL reduction which is seen to shift to higher and higher frequencies with increase in directivity angle is an additional reduction at these gas conditions and must be attributable to some other jet-related noise, such as, shockcell noise. ${ }^{\dagger \dagger}$

\footnotetext{
${ }^{\dagger}$ Recall from our previous discussion that interaction of large-scale instabilities larger than the shockcell distance with the shockcell train will result in low frequency sound that will be directed to the upstream quadrant due to the time lag between the noise generated by the upstream shock cell compared to the neighboring downstream one; on
} 

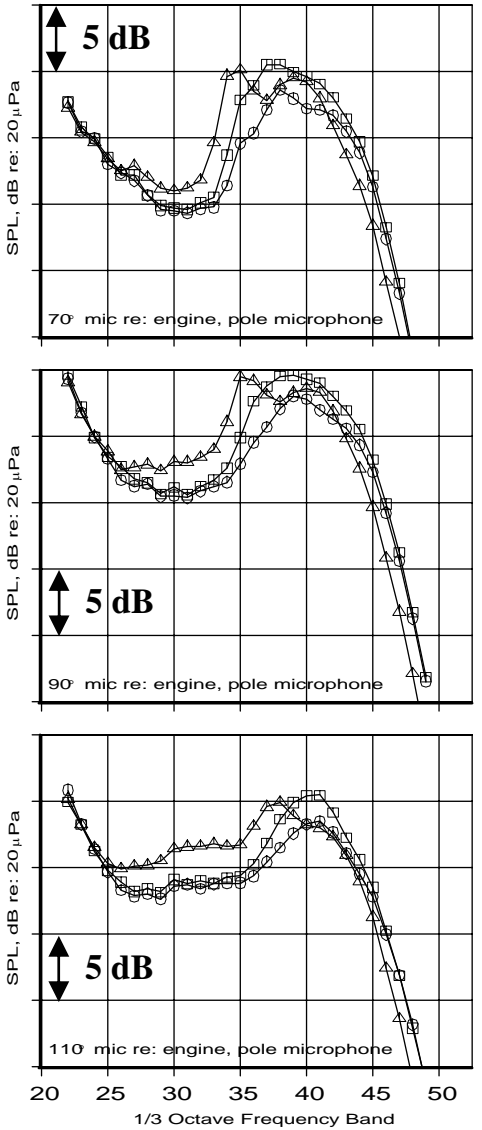

RUN 331 RUN 312 RUN 354
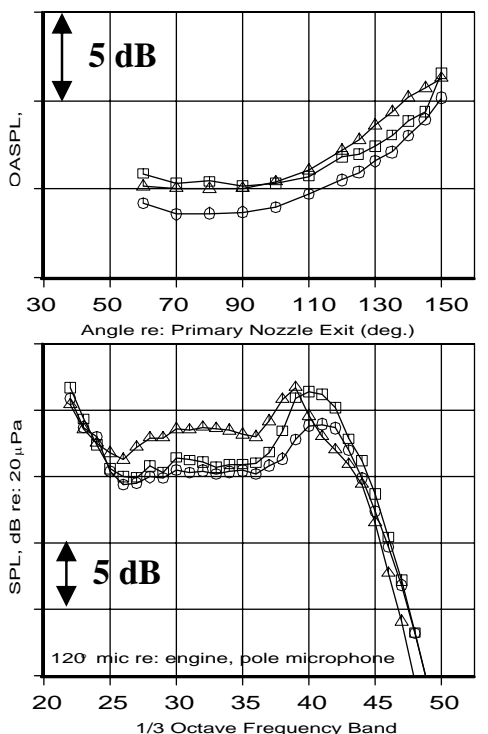
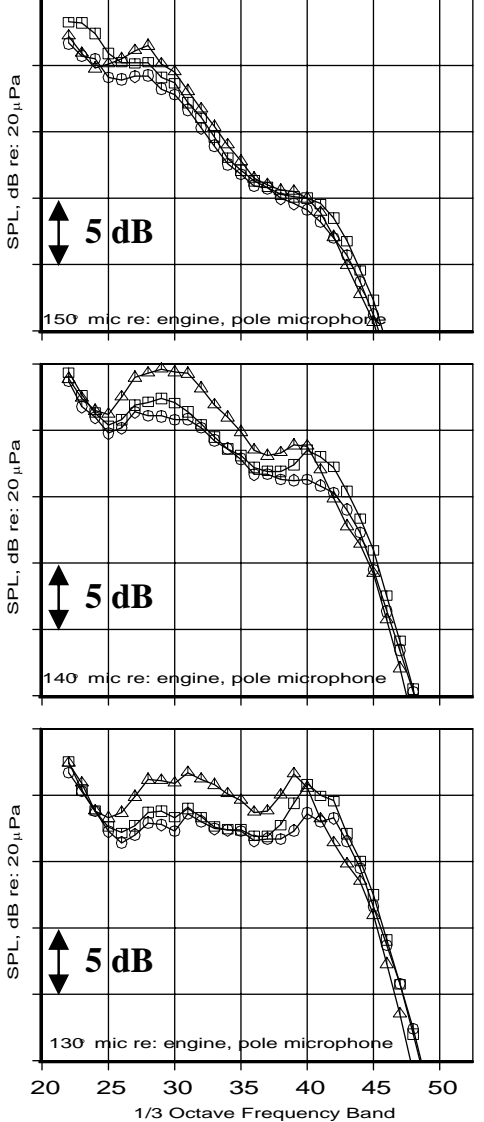

(a) SPL spectral comparison at several microphone angles.

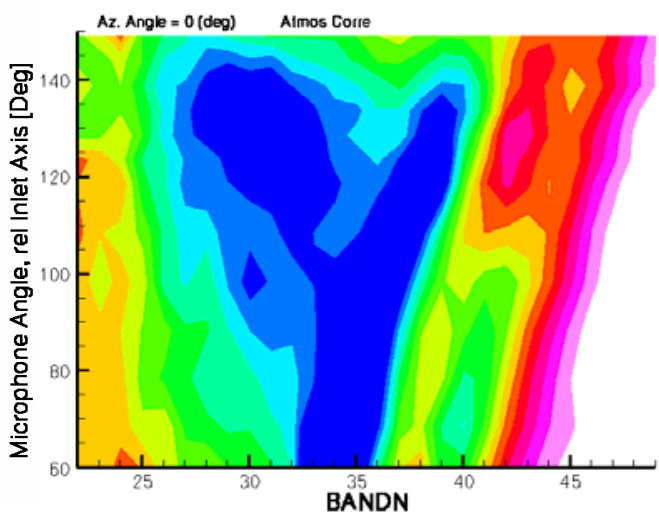

(b) SPL difference between RT and bb.
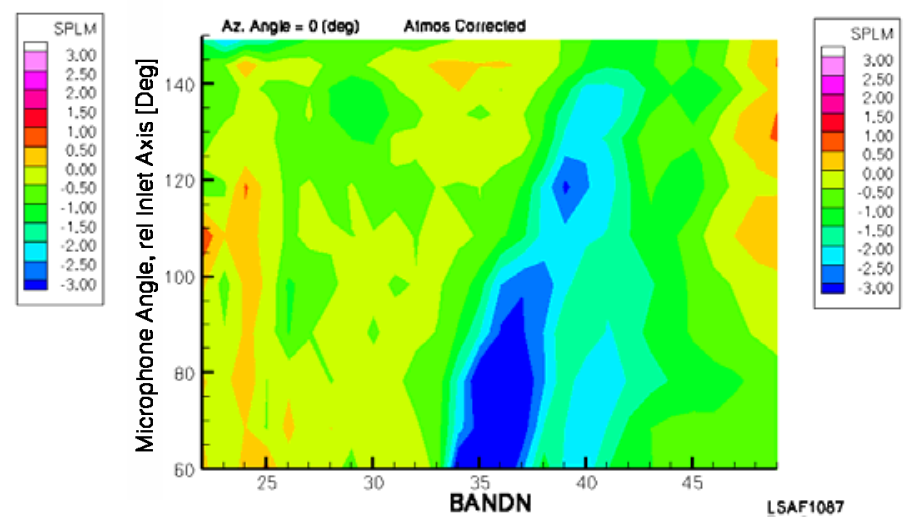

(c) SPL difference between RT and RR.

Figure 2. Far field SPL comparison "underneath" the pylon of isolated scaled model baseline (bb) nozzle (Run 354), RR-nozzle (Run 312) and RT-nozzle (Run 331) at "pseudo-cruise" conditions of $N_{P R}=2.33$, $\mathrm{NPR}_{\mathrm{p}}=1.74$ with tunnel Mach number, $\mathrm{M}_{\mathrm{wt}}=\mathbf{0 . 3 2}$.

Importantly, Fig. 2(c) shows the difference between RT-nozzle and RR-nozzle: it is striking to see that the RTnozzle is not only quieter everywhere in this spectral-directivity plane than RR, but RT is particularly effective in

the other hand, eddies having smaller wavelengths than the shock-cell spacing will be directed towards the aft arc due to the time lead between them. With convection of these wave-normals due to the tunnel flow this will typically give a pattern of peak SPL frequency which increases with directivity angle, as seen here. 
reducing the additional source of noise which is attributable to shockcell noise. The additional SPL reduction due to $\mathrm{RT}$, compared to RR, is as high as $3 \mathrm{~dB}$.

These types of scale-model results at pseudo-cruise conditions, with all its limitations and caveats, prompted us to examine the effect of the T-fan nozzle in a full-scale flight test. The primary purpose of the flight test was for studying community noise reductions with the T-fan chevron nozzle and other noise reduction devices, but studying it in cruise flight conditions was an additional bonus, difficult to simulate in current scale model wind-tunnel tests.

\section{Flight Test Aircraft, Nozzles and Instrumentation}

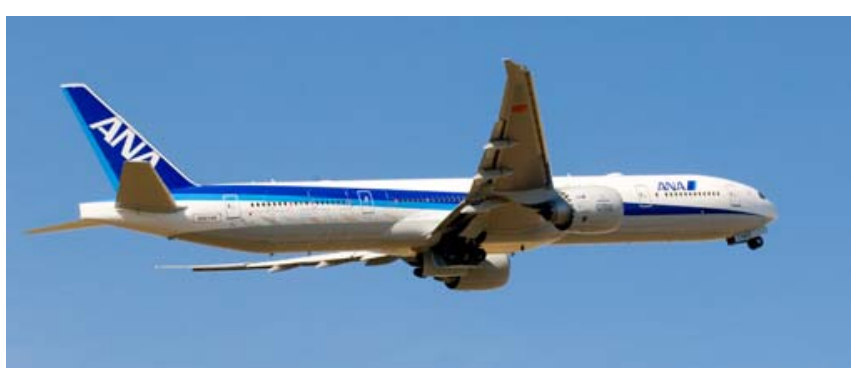

Figure 3. Flight test airplane, 777-300ER.

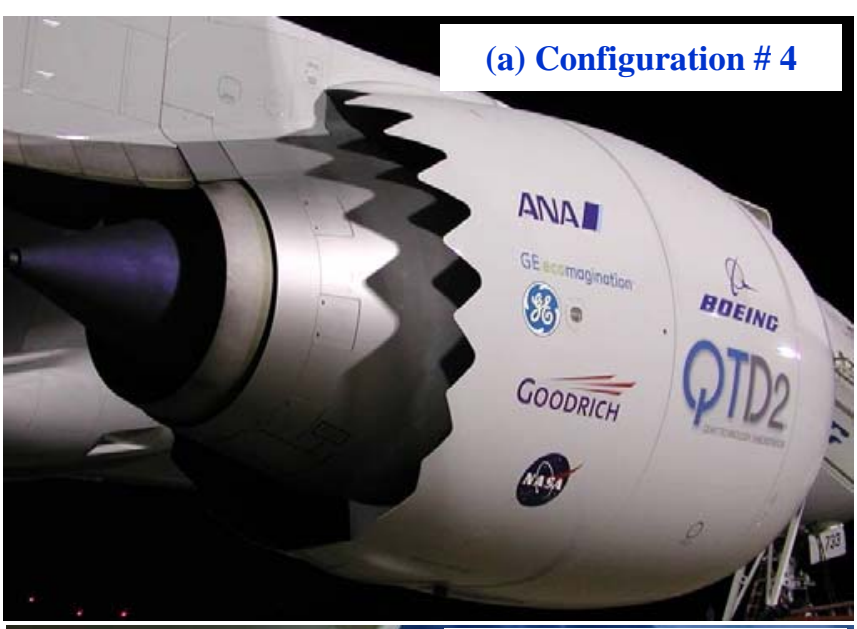

(b) Configuration \# 2

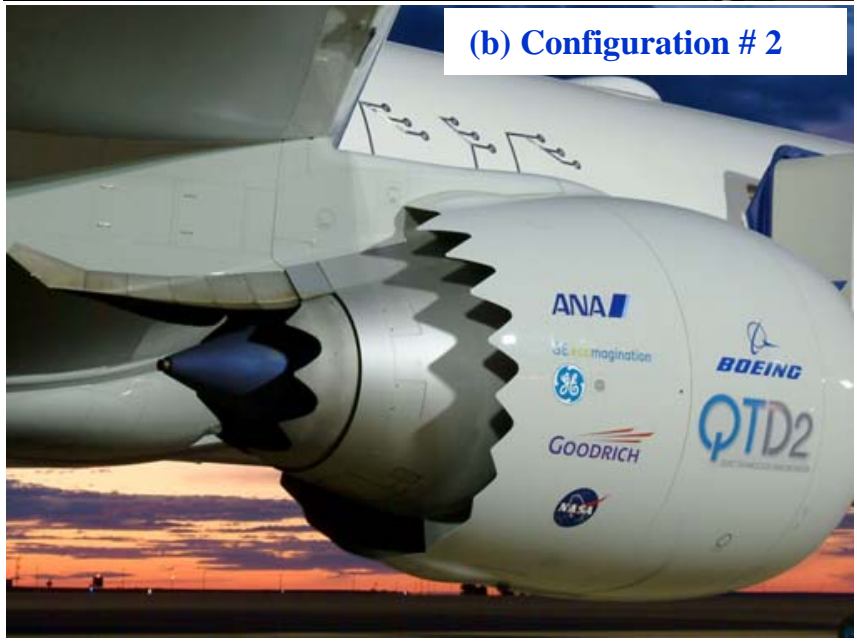

Figure 4. Flight test engine (GE90-115B) with two different nozzle modifications (a) Configuration \# 4 (bT $\mathrm{m}_{\mathrm{m}}$-nozzle), (b) Configuration \# 2, $\left(\mathrm{R}_{\mathrm{m}} \mathrm{T}_{\mathrm{m}}\right.$-nozzle).
Figure 3 shows the flight test aircraft, a Boeing 777-300ER, which is a modern, large twin-engined plane with engines under the wing. This aircraft has two GE90-115B engines which are high-thrust, high bypass ratio (7 to 8 ) engines. For this flight test, the right engine was treated for noise reduction concepts all of which have been overviewed in Herkes et al. Of most relevance to this paper are the nozzle treatments with conventional and T-fan chevrons. We discuss data from three configurations in this paper:

a) configuration 8 which has baseline simple round fan and core nozzles (without chevrons)

b) configuration 4 which has T-type fan chevrons and baseline core nozzle, and

c) configuration 2 which has above T-type fan chevrons and conventional R-type core chevrons.

Figure 4 shows configurations 4 and 2 with the chevrons. Only the right engine was modified to include the nozzle treatment (chevrons) and the left engine remained in production configuration. In this manner we can study the effect of the T-type fan chevrons, and the additional effect of conventional core chevrons on shockcell noise. The T-type fan chevrons are slightly different than the ones shown at model scale in Fig. 1 to make them flight worthy, and have 16 azimuthally varying chevrons of reducing lengths and immersions from top to bottom. We will refer to them as modified-T, or simply, $\mathrm{T}_{\mathrm{m}}$-fan chevrons, in this paper. The core chevrons are of the conventional type (azimuthally non-varying or uniform); they are 7 in number and are of constant length and immersion. Thus, in this paper, we will refer to configuration 4 as $\mathrm{bT}_{\mathrm{m}}$-nozzle (b for baseline core), and configuration 2 as $R_{m} T_{m}-$ nozzle. These chevrons were selected after a downselection of several chevron nozzles in an isolated model scale test, similar to that described in Mengle et $\mathrm{al}^{2,3,4}$. The thrust coefficient loss of $\mathrm{R}_{\mathrm{m}} \mathrm{T}_{\mathrm{m}}$-nozzle compared to the baseline nozzle, for example, was less than $0.05 \%$ at nozzle pressure ratios representative of mid-cruise conditions but with no wind-tunnel flow, as reported in the companion paper by Nesbitt et al.

The aircraft was instrumented with both exterior 


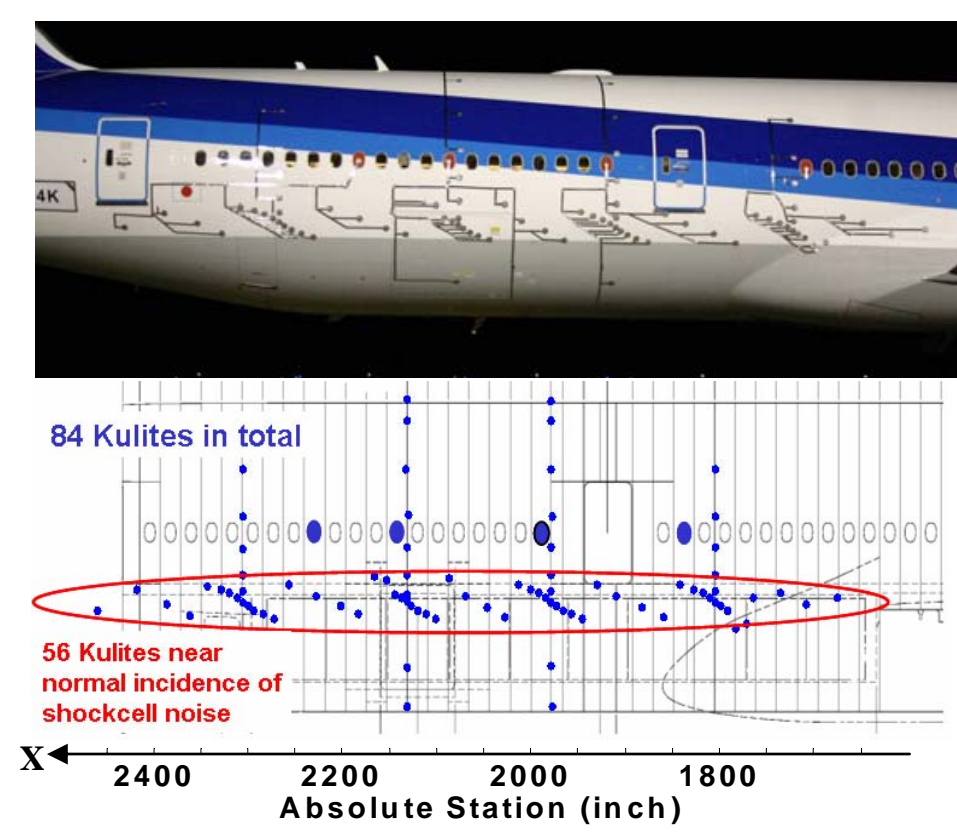

Figure 5. Unsteady pressure transducer (kulite) arrangement on the exterior of the fuselage skin on the right hand side.

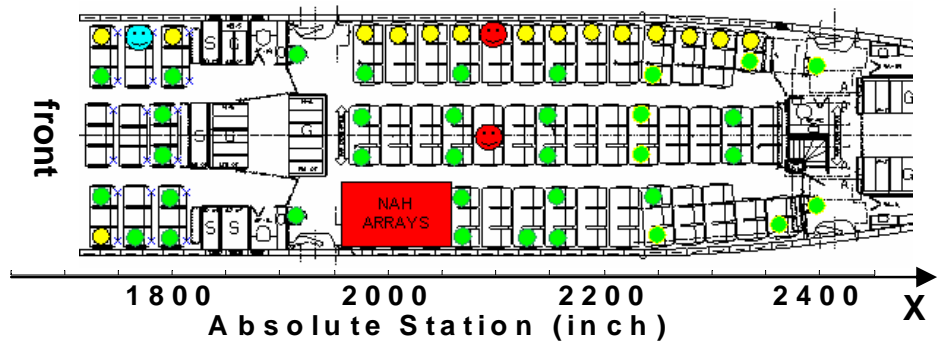

Figure 6. Interior cabin layout and microphone arrangement at head locations on the airplane seats (yellow and green circles).

unsteady pressure transducers (kulites $^{\mathrm{TM}}$ ) and interior microphones, amongst other measuring instruments. The shockcell exterior pressure field instrumentation consisted of 84 kulites. The locations of the transducers are indicated in Fig. 5 with the $\mathrm{x}$-axis indicated below the figure. The purpose of the exterior measurements included the measurement of the following properties of the shockcell noise pressure field:

- Axial distribution of the spectra,

- Circumferential distribution of the spectra,

- Coherence distribution within the four clusters,

- Shockcell source distribution.

The data presented in this paper are based on the nearly axial array of 56 transducers located near the peak region of engine noise, where the fuselage surface is closest to the engine jet plume (about 280 in.). The results reported here are only concerned with the axial distribution of the spectra. The transducers were imbedded in fairings to blend them with the fuselage surface and to minimize the effects of the transducers on the fuselage boundary layer pressure field. The transducers as well as the cables that connect them to the control and recording equipment were taped with aluminum foil tape to the fuselage surface. Transducers and cables were arranged to have a minimum impact on the boundary layer pressure field measured by adjacent sensors, in particular those downstream from a transducer.

Note that although there is some vertical separation between the kulites located near the central axis, the ratio of their vertical spread to their axial spread is about 0.043 , and they can be considered to represent the acoustic spectrum in the axial direction by neglecting their vertical separation. Assuming the shockcell train, and hence, the shockcell noise source, to be about twenty times the plume radius in axial length, the ratio of the distance of the axial kulites to the source length is about 0.325 - which makes the kulite measurements to be in the geometric near field of this non-compact source. For reference purposes the core exit plane is at an axial station of 1366 in. in relation to the x-axis shown for the transducers in Fig. 5.

The aft cabin of the airplane contained 136 economy class seats with 50 microphones mounted on seat backs as shown in Fig. 6. Each dot represents a microphone and yellow dots near the windows had in-flight visibility. Three binaural manikins (shown as faces in Fig. 6) were used to capture aurally accurate demonstration recordings. An acoustic barrier consisting of a double wall of insulated lead-vinyl blankets separated the test section from the instrumentation $\&$ analysis sections located over the wing.

\section{Test Procedure and Data Processing}

This section describes the test procedures and data processing for shockcell noise flight testing. Careful instrumentation and procedures are required to acquire quality data. This section also reviews the data processing steps to extract the shockcell noise component from the total noise signal which has considerable other flow noise components, and explains the process of normalization to account for day to day variations so that comparisons can 
be made between two configurations tested on different days and/or different times. More general information about the flight test procedure can be found in Herkes et al.

\section{A. Test procedure}

The right engine was modified to include the nozzle treatment (chevrons), as described before, and the left engine remained in production configuration and was operated at a reduced power to minimize cabin noise contamination from the non-test engine. For each of the three configurations discussed earlier (config. 8 (baseline), config. 4 (bT $\mathrm{T}_{\mathrm{m}}$-nozzle) and config. $2\left(\mathrm{R}_{\mathrm{m}} \mathrm{T}_{\mathrm{m}}\right.$-nozzle)) one or more power lines were flown. A power line is a series of engine operating conditions from a low thrust setting (producing a negligible shockcell noise component) to maximum continuous thrust (generating a significant shockcell noise component). The airplane is allowed to descend or ascend at each of these powers to maintain target flight Mach number. Therefore, the starting altitude of the airplane was chosen depending on the engine operating thrust and airplane weight to at least cross the minimum target altitude during the test condition.

The engine power settings evaluated during the test covered the full range of thrust requirements during a typical long range cruise segment of a 777 aircraft. This range extends from the lowest setting that occurs at the end of a cruise to the highest setting that occurs as the airplane climbs to the next cruise altitude. In addition to the ten test points covering this operating range, data was acquired with the engine power reduced to flight idle to gather data where the exterior pressure field of the fuselage is dominated by the boundary layer pressure field. All flight conditions were executed at a mean altitude of 35,000 feet and at an aircraft Mach number of 0.84. Low engine power settings required descending and high power settings ascending flight paths, as mentioned earlier.

All signals from the kulites and the microphones were recorded to digital tape for post flight data reduction. 36 channels were analyzed in-flight along with airplane and engine operating data. Ranges were set for the engine and airplane operating parameters and these were used to evaluate if the condition was acceptable. Immediate feedback of airplane levels was possible for acceptable conditions which could then be used to help choose further test conditions.

\section{B.Data processing}

1. Exterior Unsteady Pressure

The pressure fields measured on the exterior surface of the fuselage section aft of the wing trailing edge are the result of two primary components:

- The acoustic pressure radiating from the engine nozzles and exhaust plumes

- The mostly hydrodynamic unsteady pressure field associated with the fuselage boundary layer.

Two methods can be used to extract the engine noise component from the combined signal: the logarithmic subtraction method and the cross-spectral method.

The subtraction method makes use of the fact that the boundary layer component can be isolated by flying the aircraft at the desired Mach number on a descending glide slope with the engines throttled back to the flight idle power setting. Since the unsteady pressures in the boundary layer are proportional to the freestream dynamic pressure, their spectra can easily be scaled to actual aircraft operating conditions. This makes it feasible to extract the engine noise pressure field by subtracting spectra of the scaled boundary layer pressure field from those of the combined unsteady pressure signal. This procedure works reasonably well at high engine power settings when the two signal components are comparable.

Assuming the shockcell noise and boundary layer noise are uncorrelated, the "shockcell" noise component (which will also be considered to include the effect of shockcell noise on the boundary layer noise) at any given frequency can be written as: $p_{s c n}^{2}=p_{\text {total }}^{2}-\bar{p}_{b l}^{2}$, where $\mathrm{p}_{\mathrm{scn}}$ is the "shockcell" noise, $\mathrm{p}_{\text {total }}$ is the total or combined measured pressure, $\bar{p}_{b l}=p_{b l} \frac{\bar{q}}{q}$ is the boundary layer pressure measured during the idle power setting in the descent flight, normalized by the dynamic pressure, q, given by $2 p_{\text {amb }} M^{2}$ with a bar on top signifying a common reference condition. The ambient pressure, $p_{a m b}$, and the flight Mach number, $M$, are taken as their average values during the sampling period of 32 seconds during the test. This gives the SPL for the shockcell noise as $S P L_{\text {scn }}=S P L_{\text {total }}+10 \log \left(1-10^{-\frac{\Delta}{10}}\right)$, where $\Delta=S P L_{\text {total }}-S P L_{b l}^{\text {norm }}$, the last term in which is the SPL of the normalized boundary layer pressure, $\bar{p}_{b l}$. 
The second method makes use of the fact that the spatial coherence of the boundary layer pressure field is very limited as long as the transducers are separated laterally, normal to the streamlines by a distance of the order of one half or so of the local thickness of the boundary laye ${ }^{12}$. Because of this the cross-spectra between the signals of the two transducers is dominated by the incident acoustic pressure field and the cross-spectrum is close to the spectrum of the acoustic pressure field. This method has the disadvantage that at each location one needs at least two transducers for each measurement. Results based on this method are compromised at the very low and the high frequencies. At the very low frequency end they are contaminated by some remaining coherence in the boundary layer pressure field. At the high frequency end they are affected by the reduced coherence in the acoustic pressure field because of the finite separation of the two transducers.

We have used the subtraction method in this paper

\section{Interior Sound}

The data reduction for interior cabin measurements starts with the airplane performance time history. The time history of the altitude, Mach number, and engine setting are the most important parameters to match. Once the time window is identified for the optimal airplane performance over the condition duration, the acoustic data reduction can be completed. Typically a 32 -second integration time is used for narrowband and 1/3-octave-band analysis. Extended time windows are desirable to minimize the uncertainty due to the fluctuations in the dominant low frequency components. Corrections for Mach number, altitude, and cabin pressure can also be done.

a)

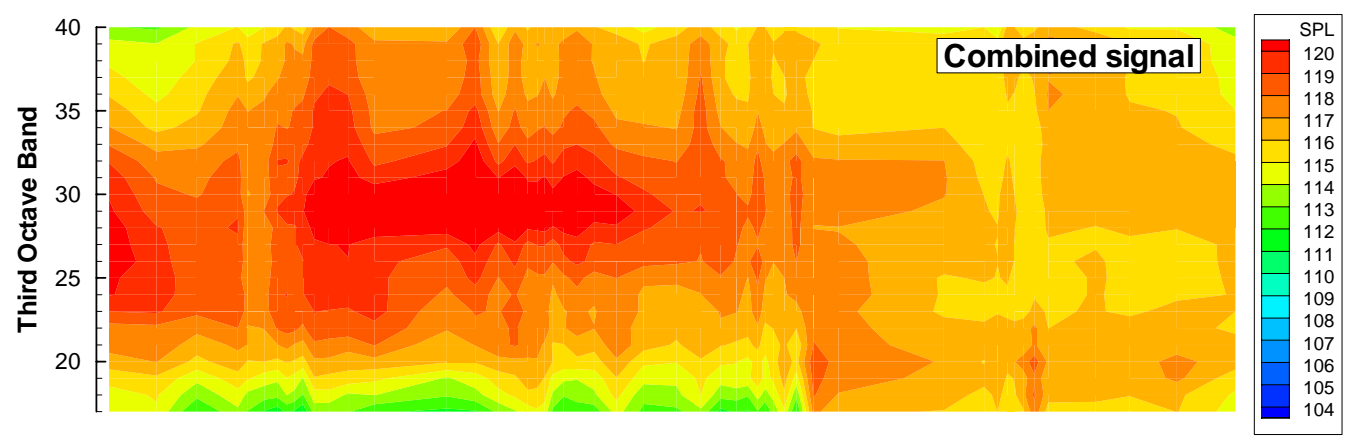

b)

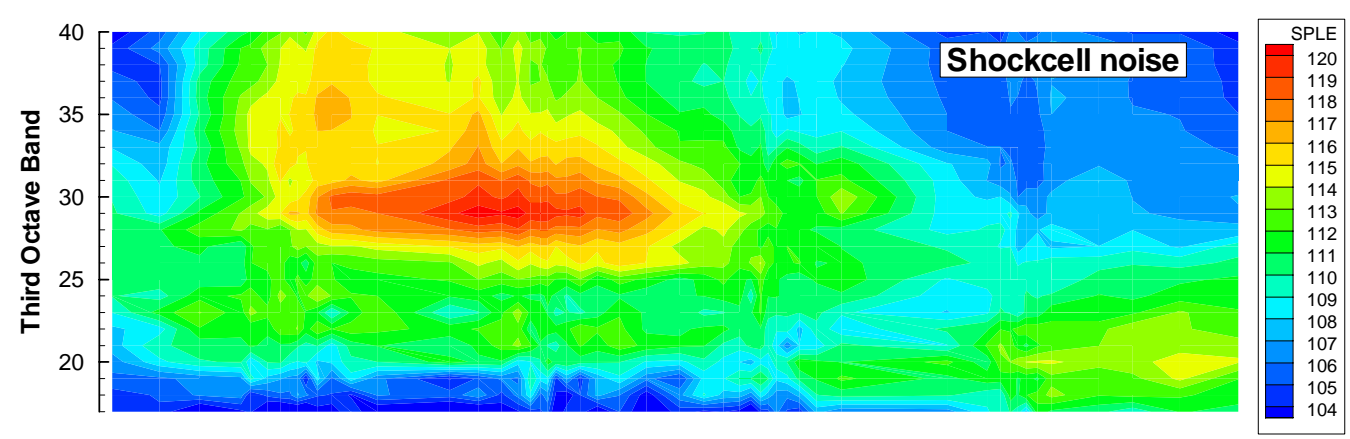

c)

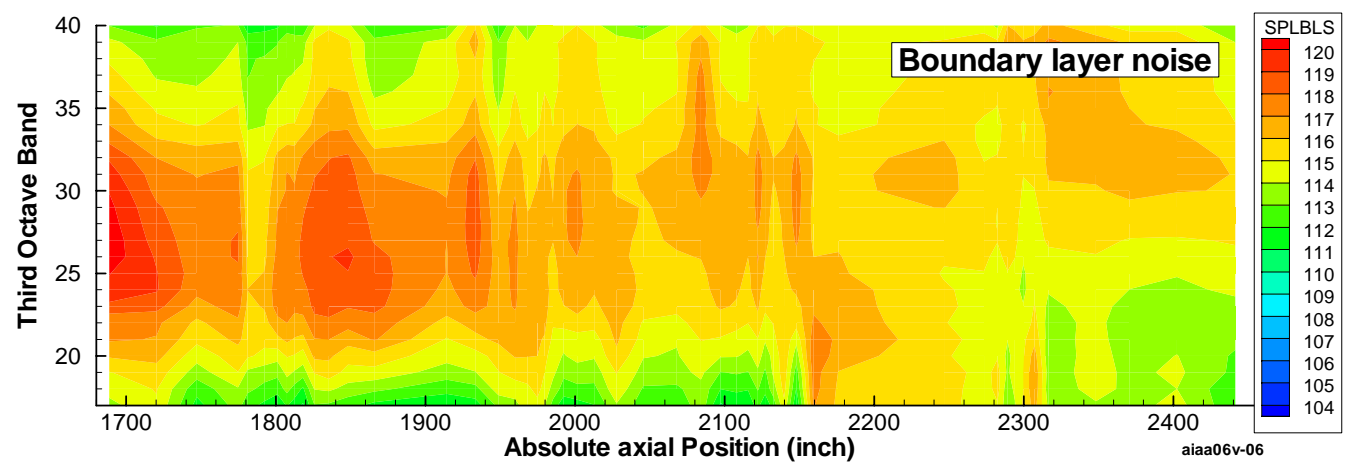

Figure 7. Example of extracting shockcell noise from the combined signal for $\mathbf{b T}_{\mathrm{m}}$-nozzle (config. 4) at an engine power setting corresponding to $\mathrm{NPR}_{\mathrm{s}}=2.46$, (a) combined signal, (b) shockcell noise, (c) normalized boundary layer noise. 


\section{Flight Test Results}
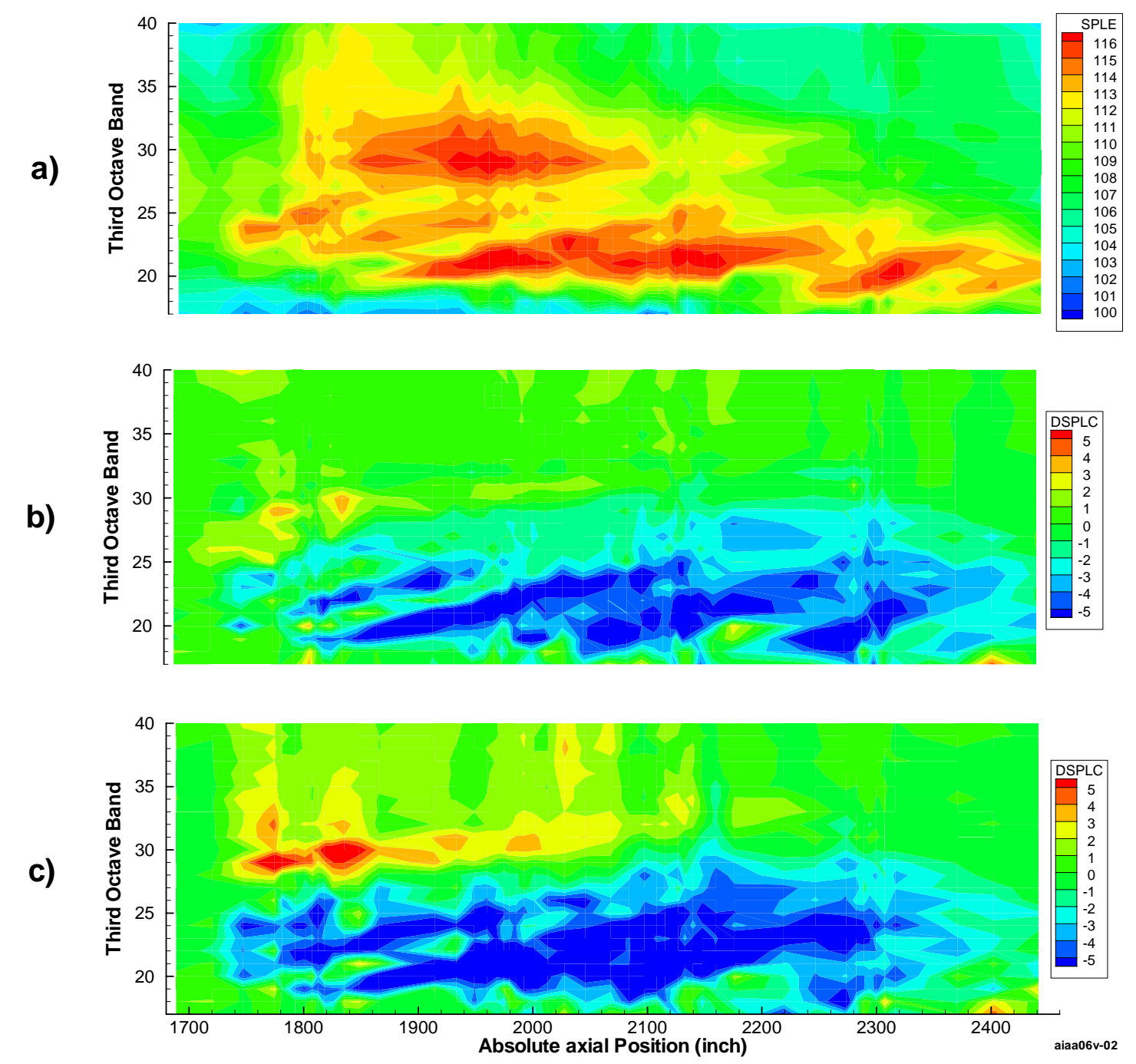

Figure 8. Comparison of SPL spectral increment in shockcell noise of the two nozzle configurations as a function of axial position at mid-cruise condition corresponding to $N_{P R}=2.37$, (a) config. 8 (baseline) SPL spectral distribution of shockcell noise, (b) config. $4\left(\mathbf{b T}_{\mathrm{m}}\right.$-nozzle) relative to baseline, and (c) config. $2\left(R_{m} T_{m}\right.$-nozzle) relative to baseline.

The flight test results are discussed in two different sections below: first, and, more importantly, for the exterior SPL spectra as obtained from the fuselage transducers, and second, for the interior microphone data. In this section we first explain the shockcell noise extraction method by an example, then analyze the differences in shockcell noise due to the presence of the T-fan chevrons alone and in combination with the conventional core chevrons, study the parametric difference on shockcell noise at different gas conditions during cruise, and finally study the effect of all these variations on internal cabin noise in terms of spectral differences near the window and spatial distribution across the whole aft cabin.

\section{A. Exterior unsteady pressure data}

All exterior data presented in this paper is based on the logarithmic subtraction method described before. The error band on the pressure transducer data is $\pm 0.3 \mathrm{~dB}$. An example of this method for extracting shockcell noise is shown in Fig. 7 for nozzle config. 4 (PAA T-fan chevrons with baseline core nozzle) at an intermediate engine power setting (both fan and core streams are supersonic at the condition shown and each would have its train of 

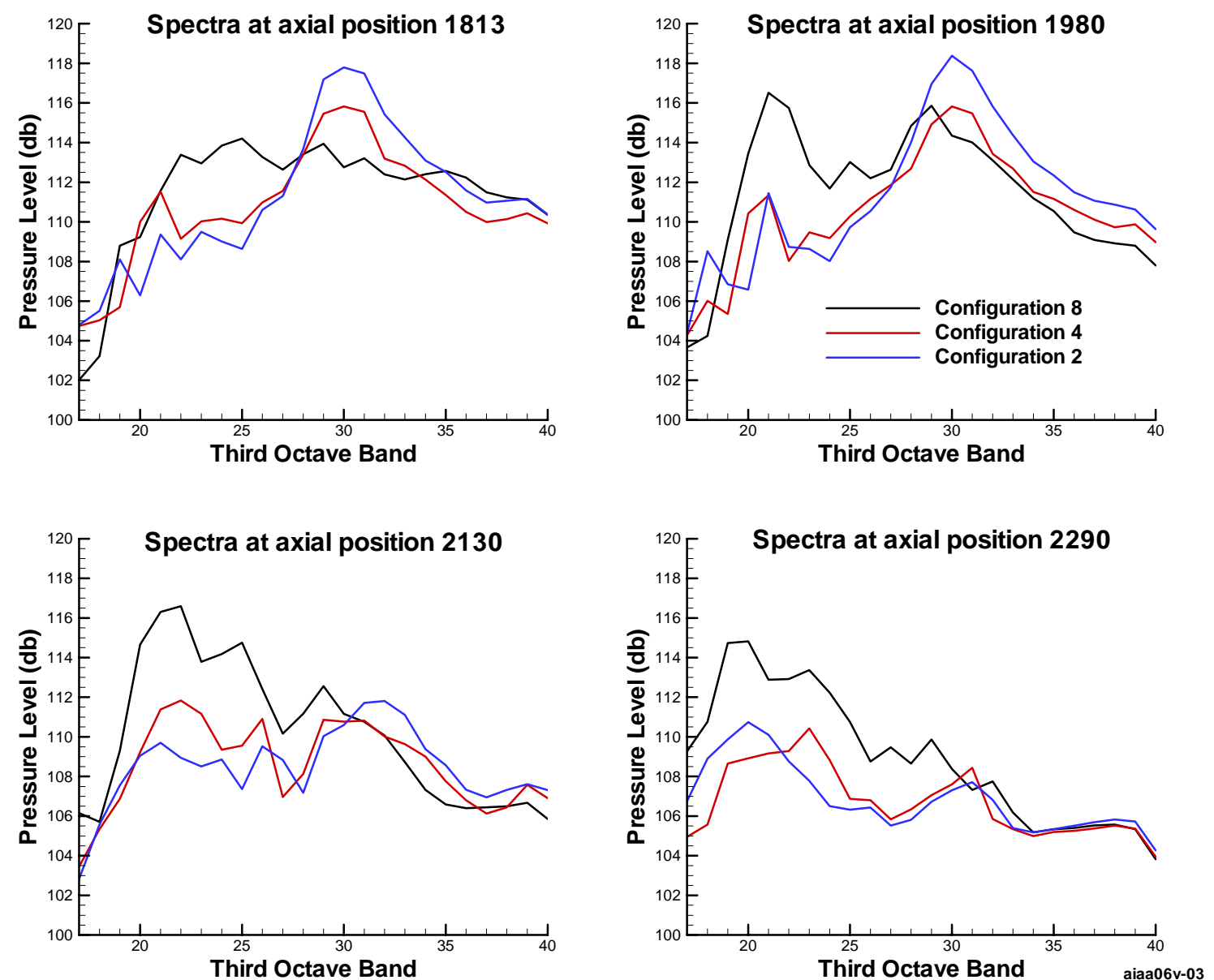

Figure 9. Comparisons of shockcell noise spectra for config. 8 (baseline), config. 4 (bT $\mathrm{m}^{-n o z z l e)}$ and config. $2\left(R_{m} T_{m}\right.$-nozzle) at four axial positions and a mid-cruise engine power setting corresponding to $\mathrm{NPR}_{\mathrm{s}}=2.37$ (same as in Fig. 8).

shock-cells). These one-third octave band spectrum maps for the combined signal, the shockcell noise and the boundary layer noise are based on the spectra of all the transducers in the axial array and are shown as a function of the axial coordinate in the aircraft frame of reference. For this particular condition, notice that the boundary layer noise distribution is quite similar to the total noise and the shockcell noise dominates at mid-bands in the relatively upstream region.

Now let us study such shockcell noise spectrum maps for the three configurations $(8,4$ and 2$)$ at conditions which typically occur at mid-cruise where the fan or secondary stream is supercritical $\left(\mathrm{NPR}_{\mathrm{s}}=2.37\right)$ and the core or primary stream is subcritical $\left(\mathrm{NPR}_{\mathrm{p}}=1.81\right)$. Figure $8(\mathrm{a})$ shows this shockcell noise pressure field for the baseline nozzle configuration. Notice the inclined peak SPL region for the lower bands (20 through 25) at stations aft of 1800 in. Each inclined peak SPL line implies that the further down the axial station is the higher the frequency of the peak SPL.

This is very reminiscent of the observation made from the scale-model test data in Fig. 2 that the peak SPL frequency shifts to higher values as the observer is placed at further and further aft angles. There is a one-to-one correspondence between the far field directivity angle in LSAF and the axial station of the kulites on the fuselage, although the latter is in the geometric near field. Also, in the flight test case, the convection of the wave-normals from the source (shockcells) is much higher due to the relatively high wind-speed $(\mathrm{M}=0.84)$ in the reference frame of the airframe or kulites; hence, these wave-normals are convected even further downstream than in the LSAF case $\left(\mathrm{M}_{\mathrm{wt}}=0.32\right)$. The multiplicity of these inclined stripes of peak SPL is believed to be from several harmonics of the 
a)
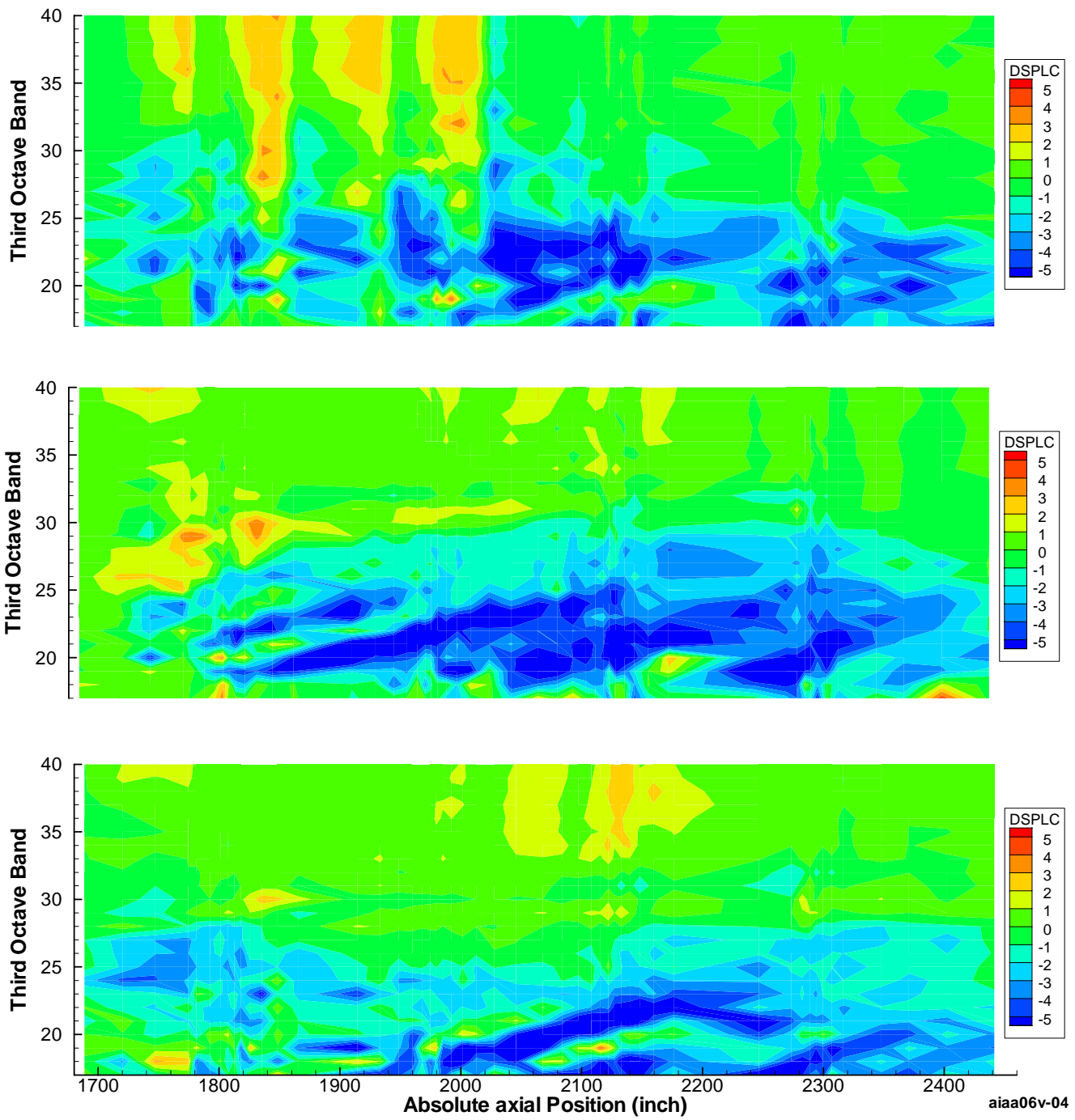

Figure 10. Effect of engine power setting at cruise on the shockcell noise spectral differences between configuration $4\left(\mathrm{bT}_{\mathrm{m}}\right.$-nozzle) and the baseline configuration with secondary nozzle pressure ratio $\left(\mathrm{NPR}_{\mathrm{s}}\right)$ of (a) 2.31, (b) 2.37 and (c) 2.46.

fundamental interaction of the large-scale instability and the shockcells, and the constructive interference pattern that is formed thereby.

In other data, not shown here due to brevity, we have also observed that peak frequencies shift to lower values with increasing velocities, Mach numbers. This is associated with the increase of the shockcell length with increasing jet Mach numbers. These two features show that the signal we are extracting from the measured total signal is, indeed, from shockcell noise.

Now consider the change in this noise component due to the T-fan nozzle alone, that is, configuration 4 at this same gas condition. Figure 8(b) shows that there is a large decrease in the low-frequency noise component associated with shockcell noise of about $5 \mathrm{~dB}$ or so, without any significant high frequency lift (HFL) in the SPL. This is one of the most important findings in this paper. Figure 8(c) shows the effect of adding the conventional core chevrons to the T-fan nozzle, config. 2. The combination of the PAA T-fan chevrons with the primary chevrons (configuration 2) seems to provide slightly higher noise reduction in the lower frequencies, but this small benefit comes with a substantial increase in the high frequencies.

Figure 9 shows a line-plot comparison of the actual third octave band spectra of the shockcell noise component associated with configurations 2, 4 and 8, shown in Fig. 8, at several axial stations for the same gas conditions as 
a)

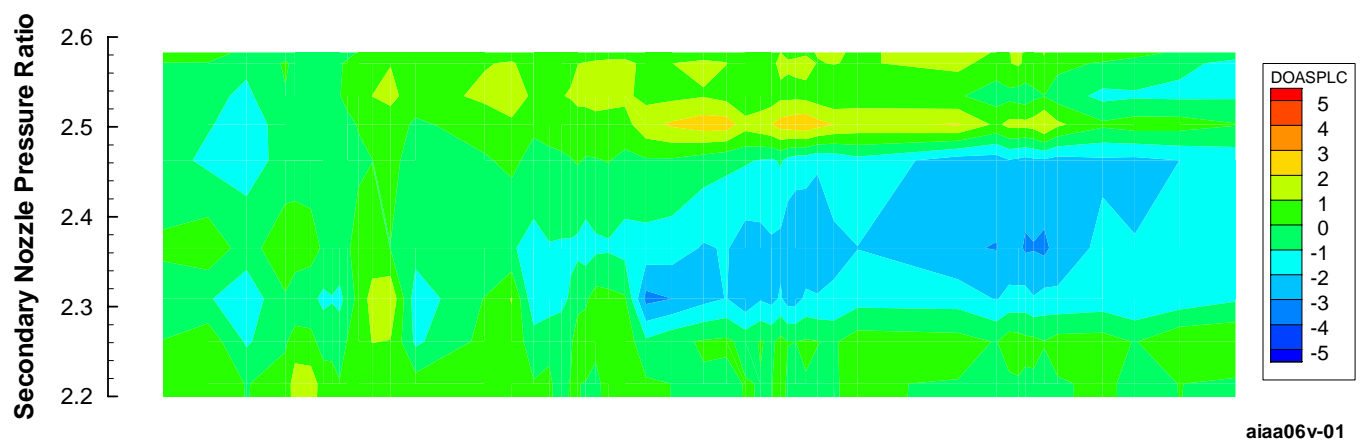

b)

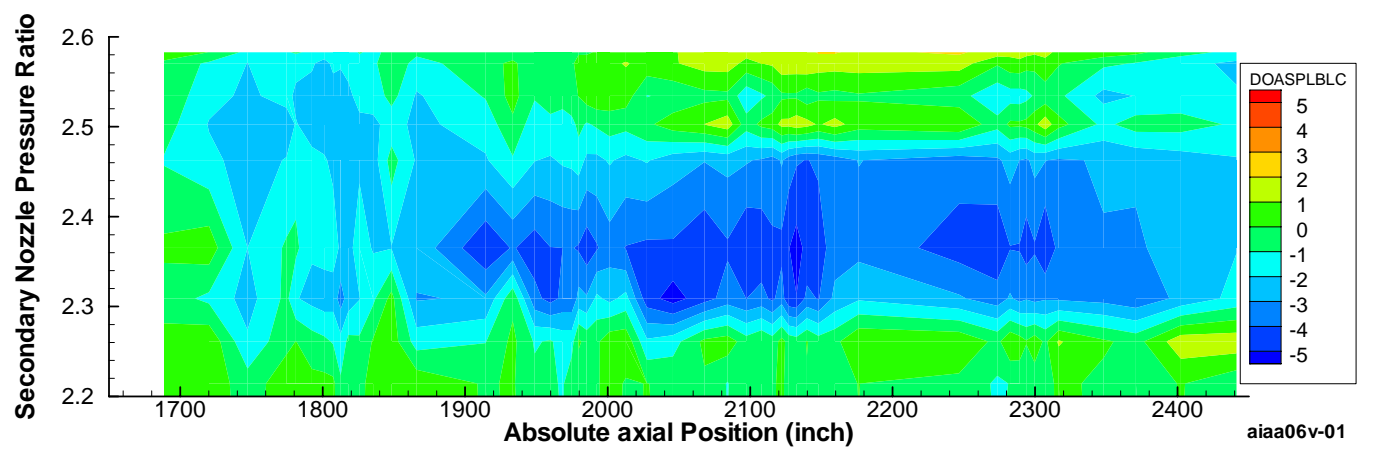

Figure 11. Effect of engine power setting on increment of OASPL of shockcell noise of config. 4 $\left(\mathrm{bT}_{\mathrm{m}}\right.$-nozzle) relative to baseline as a function of axial position and secondary nozzle pressure ratio, (a) OASPL for bands 17 to 40, (b) Band-limited OASPL for bands 18 to 25.

before. Compare these plots to Fig. 2 from scale-model tests in LSAF and notice the similarity of shifting the SPLspectra to the right for both the configurations, but also shifting it down in amplitude for the T-fan chevrons alone (config. 4). Thus, there is a lot of similarity between the LSAF data and the flight data. These plots demonstrate that the chevron configurations (config 2 and 4 ) have consistently lower noise levels in the low frequency region than the baseline nozzle. In general the PAA T-fan chevrons cause a small increase in the high frequencies to levels slightly higher than those of the baseline configuration. The combination of the PAA T-fan chevrons with the primary chevrons (configuration 2) seems to provide slightly higher noise reduction in the low frequencies, but this small benefit comes with a substantial increase in the high frequencies. This penalty, however, appears to die down at downstream stations.

Now consider the effect of the nozzle pressure ratios on the PAA T-fan chevron nozzle, config 4. Figure 10 shows spectral maps of the increments between configurations 4 and 8 at three different engine power settings $\left(\mathrm{NPR}_{\mathrm{s}}=2.31,2.37\right.$ and 2.46; the first two settings also have subcritical primary NPR but the last one has supercritical primary NPR). The data demonstrates that the T-fan chevrons cause significant reductions in the low frequency shockcell noise with minimal increases in the high frequency region. The results also indicate that this effect is present over a significant range of intermediate power settings. The vertical stripes in the high frequency region of Fig. 10(a) are associated with the difficulties to accurately define the shockcell noise when its signal is significantly lower than the boundary layer noise, a common occurrence at low power settings.

Finally, Figure 11 summarizes the effect of the T-fan chevrons on the shockcell noise pressure field of config. 4 relative to the baseline config. 8. It shows the OASPL measured by the axial array of transducers as a function of axial position and engine power setting. The engine power setting is represented by the secondary nozzle pressure ratio. Figure 11(a) depicts the true OASPL based on all bands of the third octave band spectra. The noise reductions due to the PAA chevrons are rather small. This results from the fact that the exterior pressure field includes a significant contribution by the high frequencies especially at the high engine power settings and in the forward section of the aft cabin. Figure 11(b) shows a band-limited OASPL based on the integration over bands 18 to 25 only. This parameter represents the low frequency content of the spectra, the segment of the spectrum that is difficult to attenuate with the fuselage sidewall. The results indicate that the noise reduction due to the T-fan chevrons occur primarily at the intermediate engine power settings with a small increase at the highest engine power 
settings. However during typical aircraft missions the engine operation at high power settings is limited to short time periods and most of the time is spent at the intermediate engine power settings represented by $\mathrm{NPR}_{\mathrm{s}}=2.37$.

\section{B. Interior Cabin Noise Data}
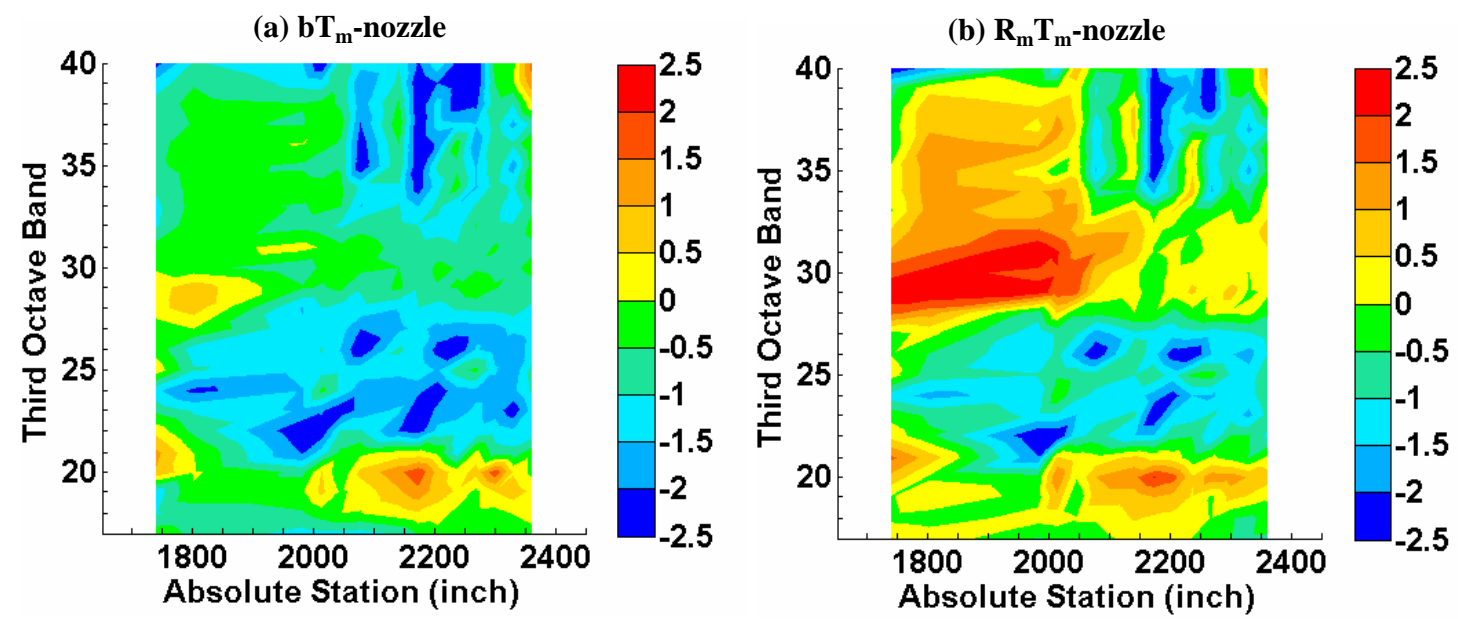

Figure 12. Comparison of difference in total SPL increment for interior window microphones, referenced to baseline configuration, at mid-cruise condition with $\mathbf{N P R}_{\mathrm{s}}=$ 2.37 for two configurations (a) config. 4 (bT $\mathrm{m}_{\mathrm{m}}$-nozzle), and (b) config. $2\left(\mathrm{R}_{\mathrm{m}} \mathrm{T}_{\mathrm{m}}\right.$-nozzle).

Figure 12 shows the microphone data for the right hand window seats, shown in Fig. 6 as yellow dots, for the two configurations studied earlier for their exterior data in Fig. 8(b) and 8(c) during the same flight. The error band on all microphone data is $\pm 0.3 \mathrm{~dB}$. Config. 4 with the T-fan chevrons alone appears to have reduced low frequency noise almost at all axial locations compared to the baseline (Config. 8) without increasing the high frequency noise, just like its exterior SPL data change from the transducers. The peak SPL reduction inside the fuselage near the window is as high as 2 to $2.5 \mathrm{~dB}$. On the other hand, Fig. 12(b) shows that the effect of adding the conventional core chevrons is to raise the high frequency noise and decrease the noise benefit at low frequencies. From this figure alone one would tend to include that the core chevrons are not needed to reduce the shockcell noise, but recall that these are just one-point designs, and generalizations are difficult.

Now, for the same Config 4 (T-fan alone), which proved to be quieter, let us study the effect of NPR. Figure 13 shows the difference in window SPL for two sets of NPRs: (i) the low powered ones $\left(\mathrm{NPR}_{\mathrm{s}}=2.26\right.$ and 2.37) in which fan $\mathrm{NPR}_{\mathrm{s}}$ is supercritical but the core $\mathrm{NPR}_{\mathrm{p}}$ is subcritical, and the high-powered ones $\left(\mathrm{NPR}_{\mathrm{s}}=2.46\right.$ and 2.53) in which both fan $\mathrm{NPR}_{\mathrm{s}}$ and core $\mathrm{NPR}_{\mathrm{p}}$ are supercritical. Exterior pressure transducer data for conditions corresponding to $\mathrm{NPR}_{\mathrm{s}}=2.37$ (Fig. 13(b)) and $\mathrm{NPR}_{\mathrm{s}}=2.46$ (Fig. 13(c)) was shown earlier (see Figs. 8 and 7). The window seat microphone data very much corresponds to the exterior transducer data with reasonable low frequency reductions of up to $2 \mathrm{~dB}$ at the $\mathrm{NPR}_{\mathrm{s}}=2.37$ condition - this gas condition also happens to be the target mid-cruise condition for this plane. At the highest $\mathrm{NPR}_{\mathrm{s}}$ shown (Fig. 13(d)), with both flows supercritical, and which occurs only during increases of altitude in the beginning phases of cruise, there is a significant increase in high frequency noise. On the other hand, at the lowest power shown (Fig. 13(a),) which occurs at the end of cruise, the difference is within $\pm 0.5 \mathrm{~dB}$ of the baseline nozzle.

Finally, Figs. 14(a) and 14(b) show the spatial distribution of the change in SPL for config. 4 in the whole instrumented aft cabin for low frequencies $(63 \mathrm{~Hz}, 125 \mathrm{~Hz}$ and $250 \mathrm{~Hz})$ and the change in OASPL compared to the baseline nozzle at the highest $\mathrm{NPR}_{s}=2.53$ (shown earlier in Fig. 13(d)). Although there is a reasonable amount of decrease in SPL near the right window at the low frequencies shown, for example, see $125 \mathrm{~Hz}$, the OASPL does not show as much decrease - at most $2 \mathrm{~dB}$ in the aft seats. This is due to the high frequency increase in SPL. When a core chevron nozzle is added to the T-fan chevrons (Config. 2) Fig. 15 shows a similar trend for the SPL and the OASPL spatial maps as we saw earlier in the exterior pressure transducer data - although there is still some low frequency benefit, there is a larger high frequency lift and the shockcell noise benefit generally reduces compared to the T-fan chevrons alone. 

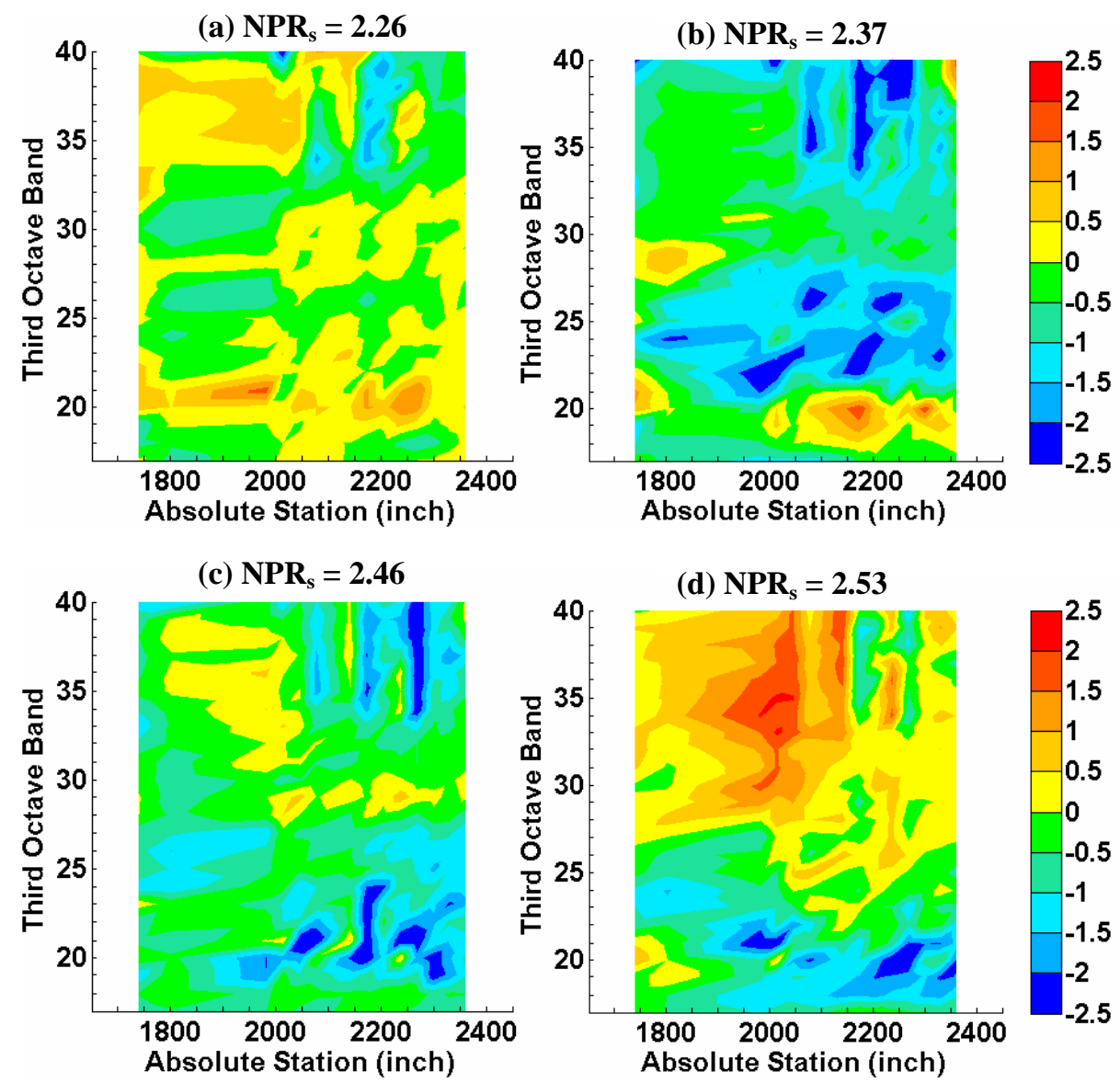

Figure 13. Variation in interior window microphone total SPL increment for config. $4\left(\mathrm{bT}_{\mathrm{m}}-\right.$ nozzle), compared to baseline configuration, with change in engine cycle conditions during cruise corresponding to different NPRs values.

\section{Concluding Remarks}

In this paper, we describe the flight test results related to shockcell noise and the corresponding interior cabin noise during cruise conditions for three different nozzle configurations on a high bypass ratio engine: baseline nozzle, PAA T-fan chevrons with baseline core nozzle, and the PAA T-fan chevrons with a conventional core chevron nozzle. The T-fan chevrons are azimuthally varying chevrons with enhanced mixing near the pylon. It is seen that the PAA T-fan chevrons alone reduce the low-frequency shockcell noise by $5 \mathrm{~dB}$ on the exterior of the fuselage without much increase in high frequencies. This is also reflected as decrease in the low-frequency cabin noise near the window of about $2+\mathrm{dB}$, but, due to slight increases in the high frequency noise in the cabin, the OASPL reduction is from $1 \mathrm{~dB}$ to $2 \mathrm{~dB}$ at best. When a conventional core chevron nozzle was added to the PAA Tfan chevron nozzle both the exterior pressure transducers and the interior cabin microphones measured slightly more low frequency reduction than the previous T-fan alone configuration, but also more high frequency increase. These trends coincide with similar types of model scale nozzles tested in LSAF.

The companion paper also describes the community noise reductions at take-off conditions in a flight test with this type of T-fan nozzle. Since scale-model results for the same nozzles earlier showed hardly any cruise thrustcoefficient loss $(<0.05 \%)$ for the PAA T-fan nozzle, it makes such azimuthally varying chevrons a viable candidate to reduce cabin noise. 


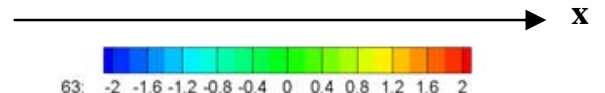

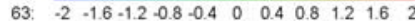

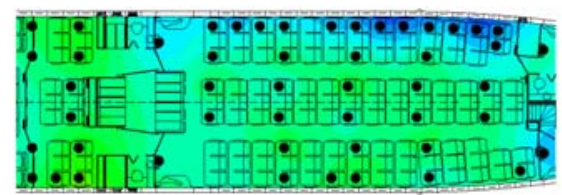

$63 \mathrm{~Hz}$
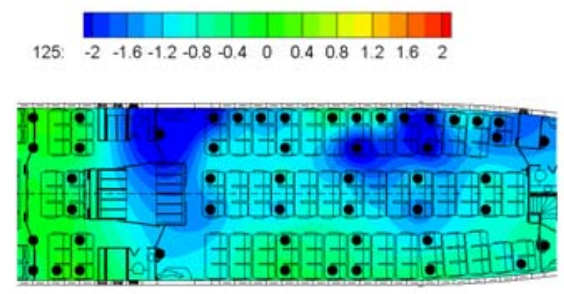

$125 \mathrm{~Hz}$
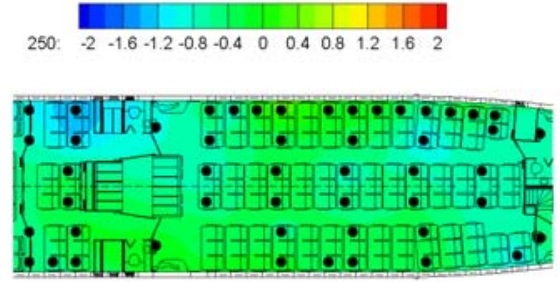

$250 \mathrm{~Hz}$

(a) SPL increment

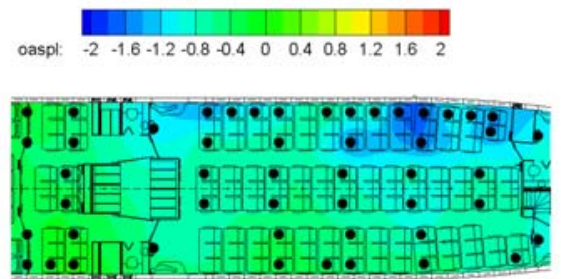

(b) OASPL increment

Figure 14. Spatial distribution of increment in (a) interior SPL at low frequencies, and (b) OASPL, for config. $4\left(\mathrm{bT}_{\mathrm{m}}\right)$ at $\mathrm{NPR}_{\mathrm{s}}=\mathbf{2 . 5 3}$.

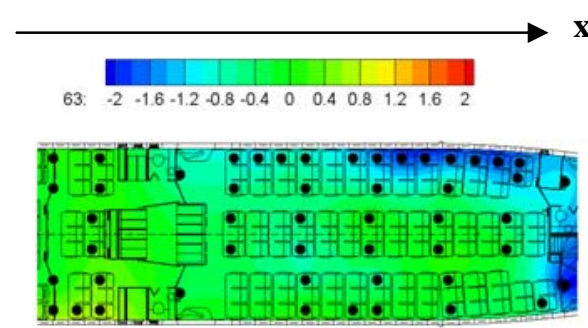

$63 \mathrm{~Hz}$
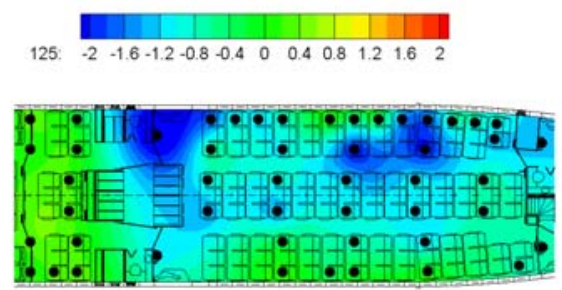

$125 \mathrm{~Hz}$
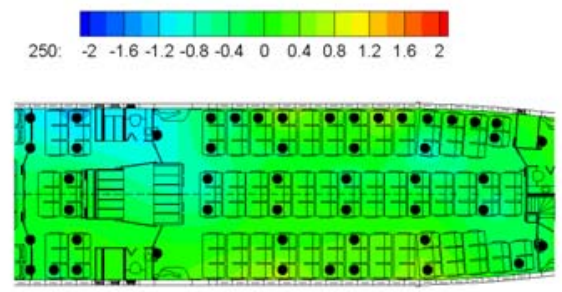

$250 \mathrm{~Hz}$

(a) SPL increment

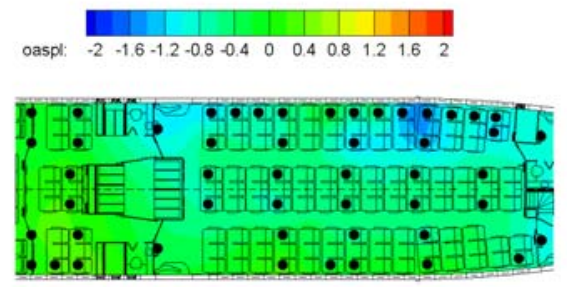

(b) OASPL increment

Figure 15. Spatial distribution of increment in (a) interior SPL at low frequencies, and (b) OASPL, for config. $2\left(R_{m} T_{m}\right)$ at $N P R_{s}=2.53$.

\section{Acknowledgments}

The Boeing-led QTD2 flight test was a joint program between Boeing, GE, NASA, Goodrich and Japan's ANA, and we sincerely acknowledge the funding, cooperation and help received from all of the partners for its great success. In particular, the PAA T-fan chevron nozzle was made under NASA Contract NNL04AA11B, Task Order: NNL05AB57T, with Dr. Russ Thomas as the technical monitor, and it was built just in time for the flight test by the dedication of the engineers and mechanics at Boeing Wichita (now, Spirit Aerospace). We would also like to particularly thank all the on-site flight test, data processing and analysis teams. 


\section{References}

${ }^{1}$ Janardan, B.A., Hoff, G.E., Barter, J.W., Martens, S., Gliebe, P.R., Mengle, V. and Dalton, W.N., “AST Critical Propulsion and Noise Reduction Technologies for Future Commercial Subsonic Engines - Separate-Flow Exhaust System Noise Reduction Concept Evaluation," NASA/CR-2000-210039, Dec. 2000.

${ }^{2}$ Mengle, V.G., Elkoby, R., Brusniak, L. and Thomas, R., "Reducing Propulsion Airframe Aeroacoustic Interactions with Uniquely Tailored Chevrons. 1. Isolated Nozzles," AIAA Paper No. 2006-2467.

${ }^{3}$ Mengle, V.G., Elkoby, R., Brusniak, L. and Thomas, R., "Reducing Propulsion Airframe Aeroacoustic Interactions with Uniquely Tailored Chevrons. 2. Installed Nozzles,” AIAA Paper No. 2006-2434.

${ }^{4}$ Mengle, V.G., Brusniak, L., Elkoby, R. and Thomas, R., "Reducing Propulsion Airframe Aeroacoustic Interactions with Uniquely Tailored Chevrons. 3. Jet-Flap Interaction,” AIAA Paper No. 2006-2435.

${ }^{5}$ Herkes, W.H., Olsen, R. and Uellenberg, S, “The Quiet Technology Demonstrator Program: Flight Validation of Airplane Noise Reduction Concepts," AIAA Paper No. 2006-2720.

${ }^{6}$ Nesbitt, E., Mengle, V.G., Czech, M., Callendar, B., and Thomas, R., "Flight Test Results for Uniquely Tailored Propulsion-Airframe Aeroacoustic Chevrons: Community Noise," AIAA Paper No. 2006-2438.

${ }^{7}$ Harper-Bourne, M. and Fisher, M. J., "The noise from shock waves in supersonic jets," Proceedings of the AGARD Conference on Noise Mechanisms, AGARD CP-131, Brussels, Belgium, pp. 1-13, 1973.

${ }^{8}$ Tam, C. K. W. and Tanna, H. K., "Shock associated noise of supersonic jets from convergent-divergent nozzles," Journal of Sound and Vibration, Vol. 81, pp. 337-358, 1982.

${ }^{9}$ Norum, T. D. and Shearin, J. G., "Shock structure and noise of supersonic jets in simulated flight to Mach 0.4", NASA TP$2785,1988$.

${ }^{10}$ Bhat, T. R. S., Ganz U. W., Guthrie A. R., "Acoustic and flow field characteristics of shockcell noise from dual flow nozzles", AIAA Paper 2005-2929.

${ }^{11}$ Long, D. F., 2005, "Effect of nozzle geometry on turbofan shockcell noise at cruise", AIAA Paper 2005-0998.

12 Bull, M.K., "Wall-pressure fluctuations associated with subsonic turbulent boundary layer flow," J. Fluid Mech., vol. 28, Part 4, pp. 719-754, 1967. 